\title{
ISO Efficiency Curves Of A -Two-Phase Hybrid Stepping Motor
}

\author{
S. Derammelaere, B. Vervisch, J. Cottyn, \\ B. Vanwalleghem, K. Stockman \\ Technical University College of West-Flanders \\ Graaf Karel de Goedelaan 5 \\ Kortrijk 8500, Belgium \\ P. Cox \\ ON Semiconductor \\ Westerring 15 \\ Oudenaarde 9700, Belgium
}

\begin{abstract}
Stepping motors are used in numerous applications because of their low manufacturing cost and simple speed control. It is well known that their energetic efficiency is low but actual values are typically not available. In this paper the influence of the control algorithm on the efficiency of the stepping motor is analyzed, measured and discussed. For a good comparison of the efficiency for the different analyzed algorithms, ISO efficiency curves are used. As the number of stepping motors installed worldwide is enormous, a few percents energy saving per stepping motor can mean a big difference in global energy use.
\end{abstract}

Keywords- Stepping Motor; ISO-efficiency curves; torque/current optimization

\section{NOMENCLATURE}

$\mathrm{C}_{\mathrm{T}} \quad$ Torque constant $(\mathrm{Nm} / \mathrm{A})$

b Friction

J Inertia $\left(\mathrm{kgm}^{2}\right)$

$t_{\text {step }}$

$t_{\text {move }}$

$\mathrm{C}_{\mathrm{emf}}$

$\mathrm{T}_{\mathrm{acc}}$

$\mathrm{T}_{\text {load }}$

$\mathrm{i}_{\mathrm{a}}$

$\mathrm{P}_{\text {out }}$

$P_{\text {in }}$

$\mathrm{P}_{\text {mech }}$

$P_{\text {loss }}$

$\mathrm{P}_{\mathrm{c}}$

$\mathrm{I}_{\mathrm{ph}, \mathrm{rms}}$

$\mathrm{I}_{\text {peak }}$

$\delta$

$\eta$

$\theta$

$\omega$

$\mathrm{R}_{\mathrm{ph}}$

$\mathrm{e}_{\mathrm{a}}$

$\mathrm{v}_{\mathrm{dc}}$

$i_{d c}$
Time between two step commands (s)

Time to move to another equilibrium point (s)

Back-emf constant (Vs/rad)

Acceleration torque $(\mathrm{Nm})$

Load torque (Nm)

Current in one phase (A)

Output power (W)

Input power (W)

Mechanical power (W)

Losses (W)

Copper losses (W)

rms current in one phase (A)

Peak current (A)

Duty cycle

Efficiency

Rotor position (rad)

Rotor speed ( $\mathrm{rad} / \mathrm{s})$

Resistance of one phase $(\Omega)$

Back-emf in one phase (V)

dc-bus voltage $(\mathrm{V})$

dc-bus current (A)
S. Derammelaere, F. De Belie, K. Stockman, L. Vandevelde

Department of Electrical Energy, Systems and Automation, Ghent University

Gent 9000, Belgium

G. Van den Abeele

PsiControl Mechatronics

K. Steverlyncklaan 13

Ieper 8900, Belgium

\section{INTRODUCTION}

Stepping motor applications are used in robotics, textile industry, domestic apparatus, etc. The simple and cheap construction, the ease of control and the open-loop capabilities of stepping motors make them very interesting in low power applications. The absence of rotor windings reduces the inertia and weight, so higher dynamics are reached. The lack of contact aging serves the reliability and mechanical ruggedness. Finally, the availability of torque at standstill makes a stepping motor to fit perfectly for diverse applications.

Despite all these advantages, drive algorithms such as open-loop full-step often result in very poor torque/current ratios.

Current stepping-motor research focuses on more advanced motion control algorithms $[1,2]$. However, in this paper the efficiencies of the basic and most popular open loop drive methodologies, full-step and half-step is analyzed. As modern drives allow to reduce the current level for full and half step profiles these possibilities are analyzed to maximize the torque/current ratio. In this way an overview of the efficiency of a two phase hybrid stepping motor is given.

As stepping motors are typically used for a broader range of torques and speeds, nominal values are not given. To present the efficiency of the motor for different control strategies, at every operating point, ISO efficiency curves are used [3, 4]. With these curves, the efficiencies of the different control strategies can be compared.

\section{STEPPING MOTOR TORQUE GENERATION}

The principle of operation of a two-phase hybrid stepper motor is illustrated in [5] and shown in Fig. 1,. The rotor is attracted by the excited phase. Fig. 2 shows the static torquerotor position curves for both phases. The solid curve illustrates the torque-position relation for an excited phase A. For a given load torque $T_{\text {load }}$, a stable operating point $p$ is reached. When a step (full step mode) command is given, phase B will be excited and the excitation of phase $A$ is removed. The dotted line illustrates the torque when phase B is excited. For the same 


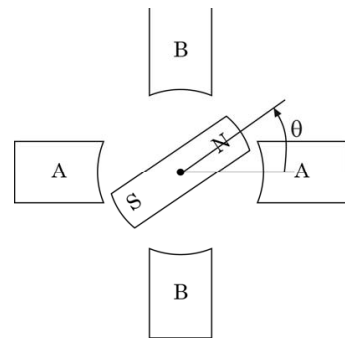

Figure 1. Simplified motor construction of a two phase

hybrid stepping motor

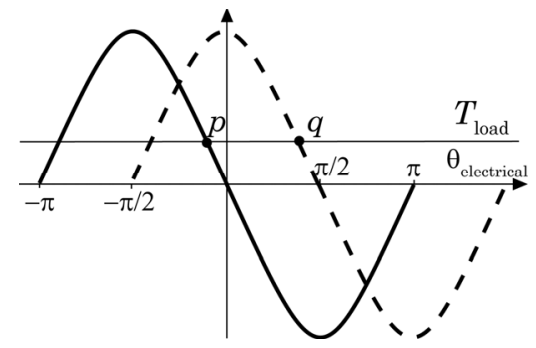

Figure 2. Static torque-rotor position curves load torque $\mathrm{T}_{\text {load }}$, the rotor moves until a new equilibrium point $q$ is reached.

As shown in Fig. 2, for an excited phase A, the maximum torque, generated by the dc-current in phase $\mathrm{A}$, is available at $\theta_{\text {electrical }}=-\pi / 2 \mathrm{rad}$. For the load torque in Fig. 2, the motor is used in position $p$. The maximum torque, related to the applied current is not required to hold the load. The torque/current ratio is not optimal resulting in high copper losses. This results in a low efficiency.

One possible solution to optimize the torque/current ratio is to reduce the current as shown in Fig. 3.a. This current reduction results in a new equilibrium point. However, because of the high number of pole pairs, this change in rotor position is in most cases not relevant. A further current reduction is not possible because of the required acceleration torque to move the rotor.

Fig 3a. shows the maximum current reduction, which is
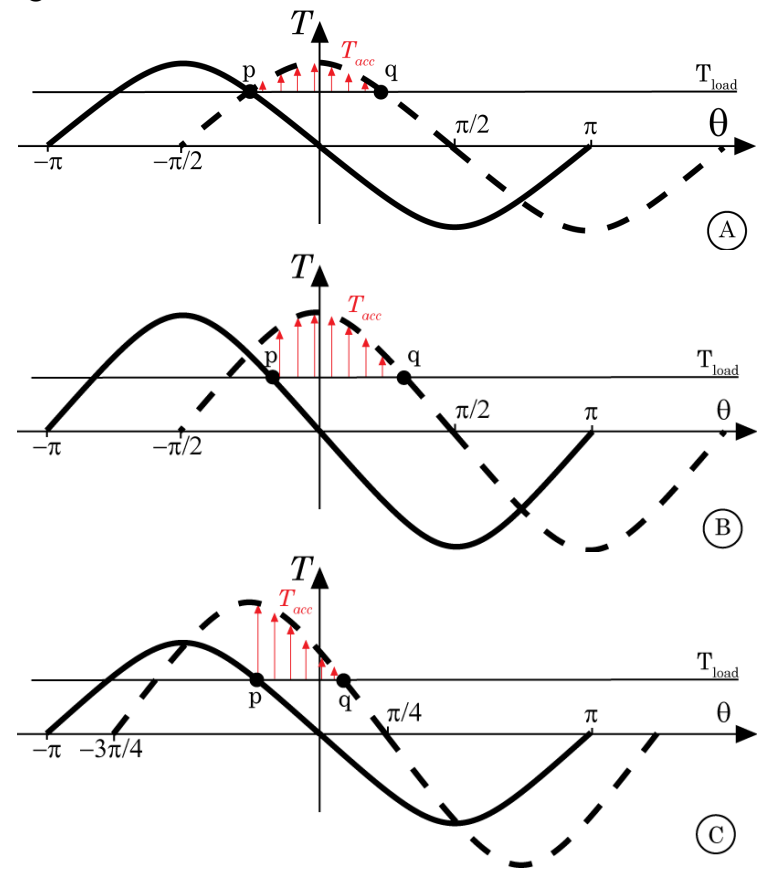

Figure 3. Reducing the current to optimize the torque/current ratio.

(A) Full-step minimum current, (B) Full-step with higher accelaration torque

(C) Half-step; Excited phase A results in the solid cuvre; Simultanious excitation of phase A and B results in the dotted line valid for a zero rotational speed. When the speed increases, the excitation time of a single phase $t_{\text {step }}$ decreases. To avoid step loss, the time $t_{\text {move }}$, during which the rotor rotates from $p$ to $q$, has to be smaller than $t_{\text {step. }}$. Therefore it can be necessary to increase the acceleration torque as in Fig. 3.b. The time $t_{\text {move }}$ is determined by equation (1):

$$
C_{T} \cdot I \cdot \sin (\theta)=T_{\text {load }}+b \cdot \frac{d \theta}{d t}+J \cdot \frac{d^{2} \theta}{d t^{2}}
$$

The constraint that $t_{\text {move }}$ has to be smaller than $t_{\text {step }}$ determines the maximum current reduction.

\section{EFFECIENCY OF A STEPPING MOTOR}

The efficiency of a stepping motor can be described as:

$$
\eta=\frac{P_{\text {out }}}{P_{\text {in }}}=\frac{P_{\text {mech }}}{P_{\text {mech }}+P_{\text {loss }}}
$$

The electric power supplied to the drive is converted into useful mechanical power as well as some electrical and mechanical losses. In general, the losses appearing in electric machines are copper losses, iron losses, friction and windage losses, and the stray load losses [6]. In a small stepping motor it is reasonable to assume that the iron losses, friction losses and stray load losses are negligible compared to the copper losses. Since the cooling is natural there are no windage losses. When only taking into account the copper losses the calculated efficiency will be a little higher than the measured efficiency.

\section{A. Copper losses}

For the representation of the copper losses the r.m.s.-value of the phase current is used. The actual phase current is discontinuous (Fig. 4). The r.m.s.-value results in a equivalent dc-current generating the same copper losses as the discontinuous phase current as shown in Fig. 4. The copper losses in a phase, using the r.m.s.-current value are given by:

$$
P_{c}=I_{p h, r m s}^{2} \cdot R_{p h}
$$

The r.m.s.-current value depends on the duty cycle:

$$
I_{p h, r m s}=I_{p e a k} \cdot \sqrt{\delta}
$$

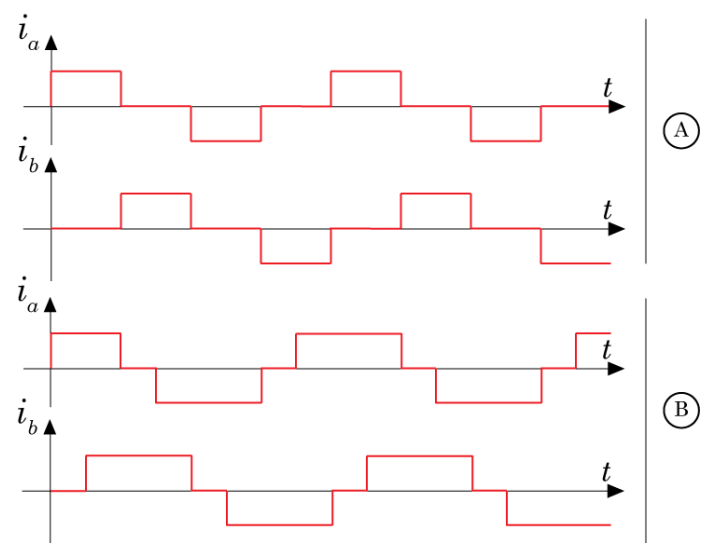

Figure 4. Full (A) and half step (B) current commands. 


\section{B. Theoretical efficiency}

Taking into account that the stepping motor has two phases, the theoretical efficiency assuming only copper losses can be written as:

$$
\eta=\frac{P_{o u t}}{P_{\text {in }}}=\frac{P_{\text {mech }}}{P_{\text {mech }}+\left(I_{p h, r m s}^{2} \cdot R_{p h}\right) \cdot 2}
$$

The peak current $I_{\text {peak }}$ for the analyzed motor is for both full and half step 5.7 A, and equals the nominal current of the motor. Each phase has a resistance of $0.5 \Omega$. With these specifications the efficiency can be mapped in an ISO efficiency curve (Fig. 5). The maps are limited by the physical torque and speed limits of the motor. The contour lines indicate a variation of one percent and the vertical color bar on the right side represents the efficiency ranging from 0 to $19 \%$.

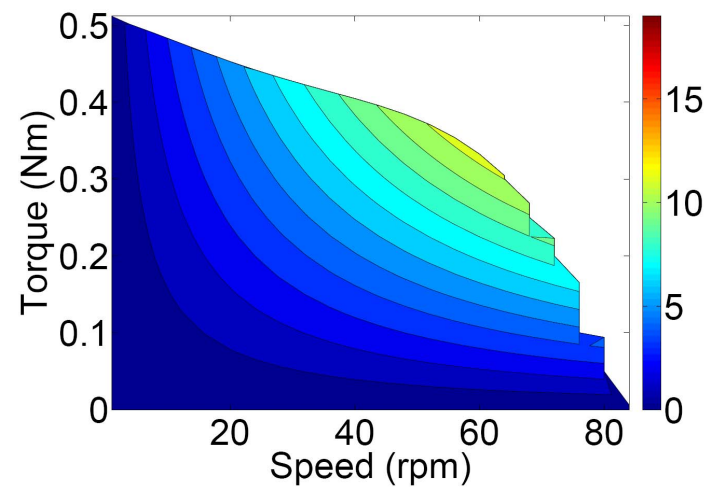

Figure 5. Theoretical efficiency (\%) curve for a stepping motor in full step.

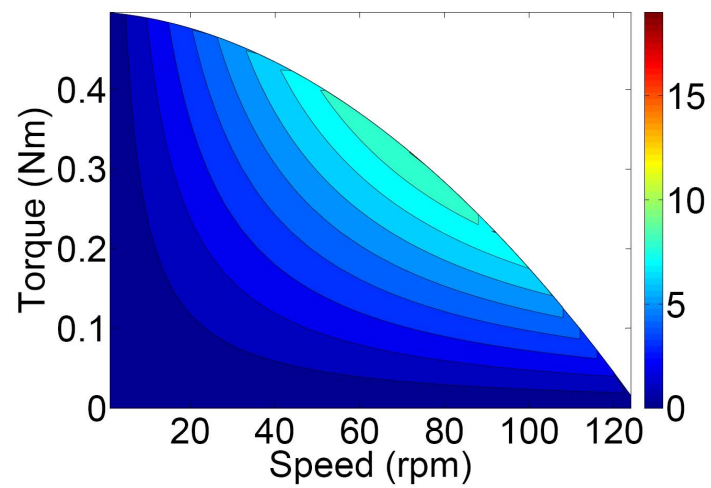

Figure 6. Theoretical efficiency (\%) curve for a stepping motor in half step.

As shown in Figs. 5-6 the efficiency of the stepping motor is very low. This is due to the high current and the relatively high phase resistance of the small motor. However, from paragraph II, it follows the desired torque can be achieved with a smaller current. By reducing the current the losses can be heavily reduced in some areas.

\section{TEST RIG}

To measure the efficiency of the stepping motor, a straight forward approach is used. The stepping motor is used to lift a known mass at a constant speed as shown in Fig. 7. In this way the load torque is perfectly known. The mechanical power can be noted as:

$$
P_{\text {mech }}=T \cdot \omega
$$

The current $i_{\mathrm{dc}}$ and voltage $v_{\mathrm{dc}}$ at the dc-bus of the stepping motor drive are measured (Fig. 8). The advantage of this approach is that the total amount of power, necessary to drive the motor can be measured. The switching losses will be included in this measurement. In this way, the power delivered to the motor can be calculated as:

$$
P_{i n}=\frac{1}{t} \int_{0}^{t} v_{d c} \cdot i_{d c} d t
$$

Then the measured efficiency is computed by:

$$
\eta=\frac{P_{m e c h}}{P_{i n}}
$$
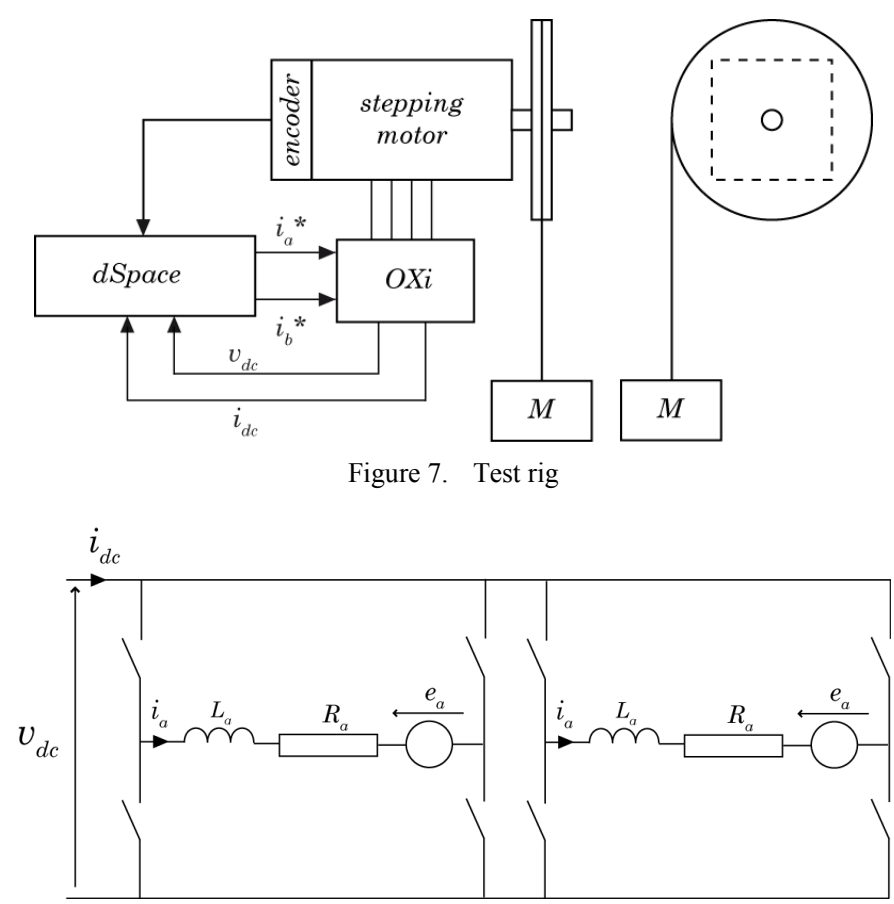

Figure 8. Electric scheme and measurements

\section{MEASUREMENTS}

Measurements are made at 13 different speed values and for 11 different load torque values resulting in a matrix of 143 measurement points. The rotor speed is chosen in a way that resonance is avoided [7]. The physical limits of the motor are the torque and rotor speed at which the motor is not able to lift the mass. These limits, clearly visible in the ISO-efficiency curves, are inherent to the mechanical parameters of the test bench, such as inertia, and will vary compared to other applications. 


\section{A. Nominal current}

First, the motor is driven with nominal current. The ISO efficiency curves for full and half step are shown in Figs. 9-10. The overall efficiency is lower than expected in the theoretical approach, especially at the higher speeds. The maximum deviation between the theoretically calculated -and measured efficiency is $1.4 \%$. This can be due to iron losses, friction losses and stray load losses. Furthermore, the currents generated by the drive are not exactly square wave. As a result, (4)is an approximation of the computed r.m.s.-value of the current.

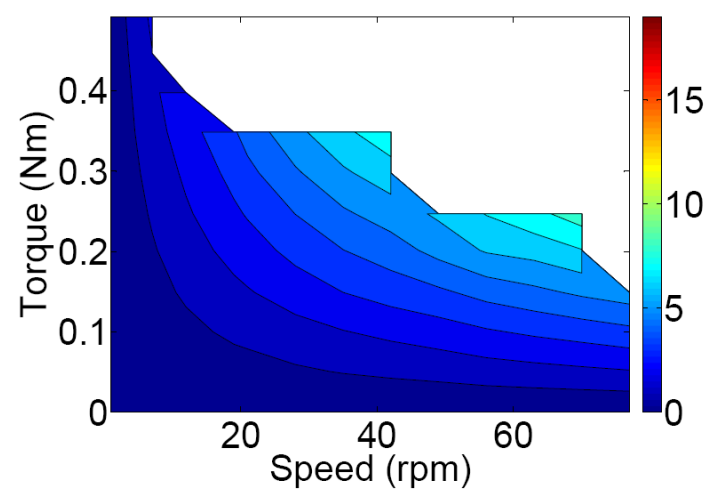

Figure 9. Measured efficiency (\%) curve for stepping motor in full step

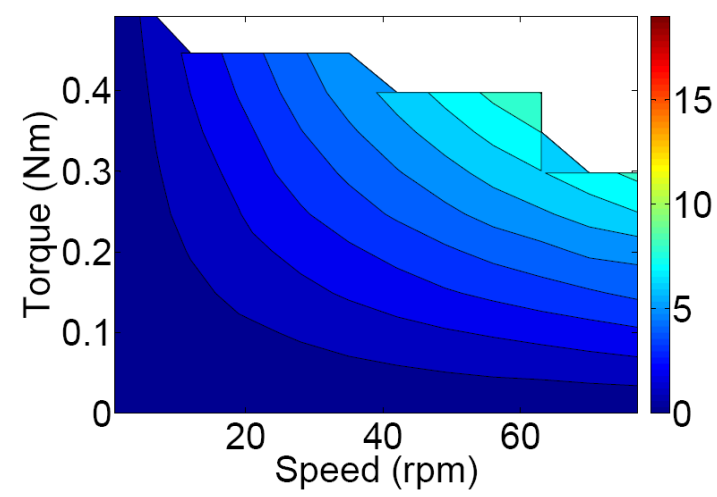

Figure 10. Measured efficiency (\%) curve for stepping motor in half step

Fig. 11 shows the currents in a single phase for different speeds. These figure show that current overshoot and oscillation occur when the current converges to zero. These oscillations increase the r.m.s.-value of the current. At low speeds, this ripple can be neglected as its duration is small compared to the excitation time $t_{\text {step }}$. However, at higher speeds it becomes more important. The increased r.m.s.-current produces higher copper losses compared to the theoretical approximation. Optimization of the current control algorithm could reduce these additional losses.
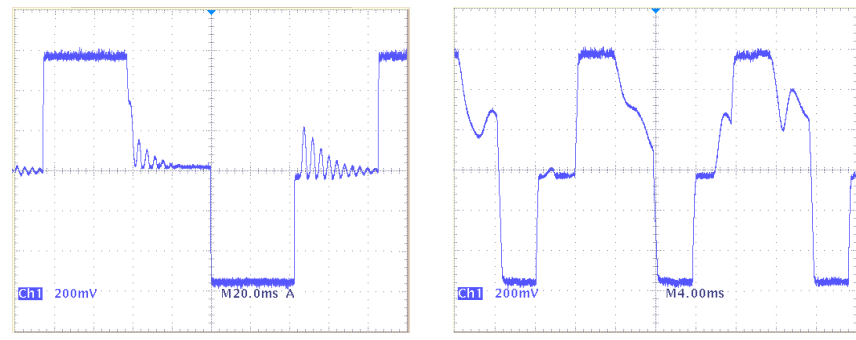

Figure 11. Current in one phase at $7 \mathrm{rpm}$ (left) and at $77 \mathrm{rpm}$ (right) (100mV/A)

\section{B. Current reduction}

To measure the efficiency at more optimal current levels, the current for every torque/speed operating point is reduced as low as possible, meaning the lowest current at which the motor is still able to lift the mass with a constant speed. Of course, this implies that there is no margin for speed or torque disturbances. Measurement results are shown in Figs. 13 and 14.

The impact of current reduction on the efficiency is maximized at half the maximum speed and torque. At low speed there is also a significant current reduction, but the losses stay relatively high compared to the useful mechanical power, resulting in a low efficiency. As shown in Fig. 3, at high speed, the torques must be high enough to accelerate the machine. As a result the margin for current reduction is low. The same reasoning also holds for high load torques.

When taking a closer look at Fig. 3.c it seems that the current reduction can be higher at half step. The necessary acceleration torque is smaller, because the distance between the two equilibrium points $p$ and $q$ is smaller with respect to full step control.

Indeed, in Fig. 13 the area of the current reduction is much larger resulting in a higher efficiency increase. Fig. 13 shows better efficiencies than Fig. 14. From Figs. 9, 10, 12 and 13 it follows that the half-step drive methodology will only lead to a higher efficiency when the current reduction is applied.

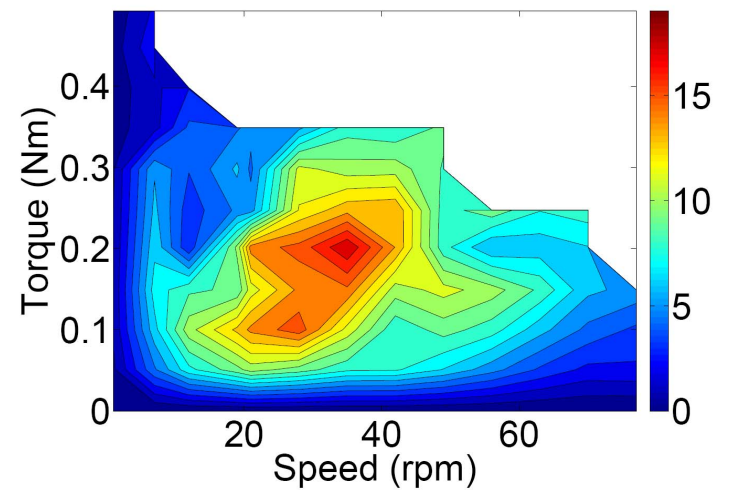

Figure 12. Measured efficiency (\%) curve for a stepping motor with full-step control and with current reduction 


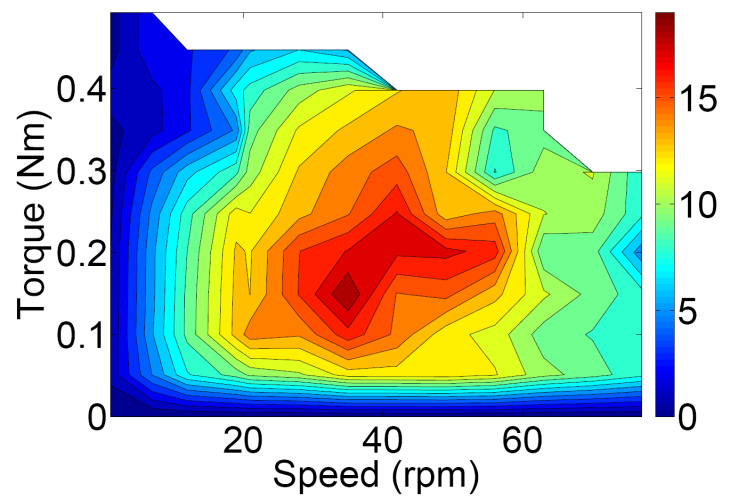

Figure 13. Measured efficiency curve (\%) for a stepping motor with half-step control and current reduction

E.g. for half-step control at $35 \mathrm{rpm}$ and with a load torque of $14.89 \mathrm{Ncm}$ the efficiency is only $2.1 \%$. By reducing the current to $23 \%$, the efficiency can be increased up to $18.9 \%$.

\section{CONCLUSIONS}

For both full- and half-step control of a two-phase hybrid stepping motor a detailed overview of the efficiency is given by using ISO-efficiency curves. These curves are obtained by a theoretical approach and through measurements.

The bulk of the stepping motor applications are driven in open loop, with maximum current to avoid step loss. These drive strategies result in very poor efficiency. To increase the efficiency the current can be reduced. The possible current reduction is a function of the operating point in the torquecurrent area. Furthermore, the maximum reduction of the current implies that there is no margin left for torque or speed disturbances. However the efficiency can be seriously increased, up to 9 times better, by this current reduction approach, which is achievable on most basic stepping motor drives.

To perform the measurements the current level was reduced until step loss occurs. An algorithm which predicts the possible current reduction could be implemented based on the mechanical equation (1). Solving this non-linear differential equation would result in a high computational cost and should be performed offline.

\section{REFERENCES}

[1] H. Gao, et al., "Maximum torque/current control of 2-phase hybrid stepping motor," in Electric Machines and Drives Conference, 2003. IEMDC'03. IEEE International, 2003, pp. 1781-1786 vol.3.

[2] L. Weijie and Z. Zhuo, "Simulation and Experiment of Sensorless Direct Torque Control of Hybrid Stepping Motor Based on DSP," in Proceedings of the 2006 IEEE International Conference on Mechatronics and Automation, 2006, pp. 2133-2138.

[3] S. Williamson, et al., "Comprehensive drive train efficiency analysis of hybrid electric and fuel cell vehicles based on motor-controller efficiency modeling," IEEE TRANSACTIONS ON POWER ELECTRONICS, vol. 21, pp. 730-740, MAY 2006.

[4] C. B. Rasmussen, et al., "Design and efficiency comparison of electric motors for low power variable speed drives with focus on permanent magnet motors," in Electrical Machines and Drives, 1995. Seventh International Conference on (Conf. Publ. No. 412), 1995, pp. 428-432.

[5] C. Kuert, et al., "New method for dynamic modeling of hybrid stepping motors," in Industry Applications Conference, 2002. 37th IAS Annual Meeting. Conference Record of the, 2002, pp. 6-12 vol.1.

[6] V. Raulin, et al., "Modeling of losses in switched reluctance machines," IEEE TRANSACTIONS ON INDUSTRY APPLICATIONS, vol. 40, pp. 1560-1569, NOV-DEC 2004.

[7] M. Bodson, et al., "Spontaneous speed reversals in stepper motors," IEEE Transactions on Control Systems Technology, vol. 14, pp. 369-373, Mar 2006. 